\title{
Bell polynomials approach for two higher-order KdV-type equations in fluids
}

\author{
Yunhu Wang, Yong Chen* \\ Shanghai Key Laboratory of Trustworthy Computing, East China Normal University, Shanghai 200062, People's Republic of China
}

\begin{abstract}
The present paper investigates the higher-order Sawada-Kotera-type equation and the higher-order Lax-type equation in fluids. The Bell polynomials approach is employed to directly bilinearize the two equations. For the Lax-type equation, bilinear Bäcklund transformation, Lax pair, Darboux covariant Lax pair and infinitely many conservation laws are obtained by means of binary Bell polynomials. Moreover, based on its bilinear form, $N$-soliton solutions are also obtained. For the Sawada-Kotera-type equation, with the help of the Riemann theta function and Hirota bilinear method, its one periodic wave solution is obtained. A limiting procedure is presented to analyze in detail the relations between the one periodic wave solution and one soliton solution.
\end{abstract}

Keywords: Bell polynomials, Hirota bilinear method, Sawada-Kotera-type equation, Lax-type equation.

PACS: 02.30.Jr, 05.45.Yv.

\section{Introduction}

As is well known, integrability of the nonlinear evolution equations (NLEEs) plays an important role in soliton theorey, which can be regarded as a pretest and the first step of its exact solvability. Among the properties that can characterize the integrability of NLEE are the bilinear representation, Bäcklund transformation (BT), Lax pair, infinitely many conservation laws, infinite symmetries, Hamiltonian structure, Painlevé test and so on. It is well known that the Hirota bilinear method [1-6] enables ones to obtain the bilinear form and bilinear BT for a given NLEE, meanwhile the bilinear BT can be directly linearized to associated Lax pair. Thus, the key of the Hirota bilinear method to construct bilinear BT, Lax pair and infinitely many conservation laws is to transform the given NLEE to corresponding bilinear form. However, the construction of bilinear form by using Hirota bilinear method is not as one would wish. It relies on a particular skill by choosing suitable variable transformations, such as rational transformation, logarithmic transformation, etc, but there is no general rule to find these transformations.

In recent years, the Bell polynomials linked with Hirota bilinear operator, are found to play an important role in the characterization of bilinearizable equations and the relationship between the integrability of a NLEE and the Bell

\footnotetext{
${ }^{*}$ Corresponding author: Tel.: +862162224199.

Email address: yychen18@hotmail.com (Yong Chen) 
polynomials [7-11]. In terms of the Bell polynomials approach, one may obtain, on one hand, such results as the Bell polynomials expression (in the $P$ or $\mathscr{Y}$-polynomials form), Bell polynomials type BT and Lax pair, and on the other hand, the connection between the Bell polynomials and Hirota bilinear method can be revealed, namely, the Bell polynomials expression can be cast into the bilinear form, and the Bell polynomials type BT can be mapped into the bilinear BT. Then, both the Bell polynomials type BT and bilinear BT can lead to the corresponding Lax pair. Moreover, with the help of the gauge transformation, Darboux covariant Lax pair which can be used to construct the higher-order members of the given equation can also be obtained. In Refs. [12, 13], Fan developed Bell polynomials approach to nonisospectral and variable-coefficient nonlinear equations. In Refs. [14, 15], Fan further developed classical Bell polynomials into super version. In Ref. [16], Ma systematically analyzed the connection between Bell polynomials and new bilinear equations.

The KdV equation arises as an approximate equation governing the weakly nonlinear long waves where the first order nonlinear and dispersive terms are retained and in balance [17]. If the second-order terms are retained, the following extended Korteweg-de Vries (eKdV) equation is given in the form [17]

$$
u_{t}+u_{x}+\alpha\left(6 u u_{x}+u_{3 x}\right)+\alpha^{2}\left(c_{1} u^{2} u_{x}+c_{2} u_{x} u_{2 x}+c_{3} u u_{3 x}+c_{4} u_{5 x}\right)=0,
$$

where $\alpha \ll 1$ is a non-dimensional measure of the small wave amplitude relative to depth, and $c_{1}, c_{2}, c_{3}$ and $c_{4}$ are the coefficients of the higher-order terms. Eq. (1.1) describes the evolution of steeper waves of shorter wave-length than the KdV equation does [18]. Under the condition $c_{1}=45 c_{4}, c_{2}=c_{3}=15 c_{4}$ and $c_{1}=30 c_{4}, c_{2}=20 c_{4}, c_{3}=10 c_{4}$, eKdV equation (1.1) leads to a Sawada-Kotera-type equation [19]

$$
u_{t}+u_{x}+\alpha\left(6 u u_{x}+u_{3 x}\right)+\alpha^{2} c_{4}\left(45 u^{2} u_{x}+15 u_{x} u_{2 x}+15 u u_{3 x}+u_{5 x}\right)=0,
$$

and a Lax-type equation [19]

$$
u_{t}+u_{x}+\alpha\left(6 u u_{x}+u_{3 x}\right)+\alpha^{2} c_{4}\left(30 u^{2} u_{x}+20 u_{x} u_{2 x}+10 u u_{3 x}+u_{5 x}\right)=0 .
$$

In present paper, our attention will be paid to the Bell polynomials approach, Hirota bilinear method and Riemann theta function method to perform the analytic study on Eq. (1.3) and Eq. (1.2).

Based on the above analysis, the main contexts of this paper will be organized as follows: In Sec. 2, we will bilinearize Eq. (1.3) and Eq. (1.2) with the help of the Bell polynomials approach. For Eq. (1.3), we introduce an auxiliary variable to construct its bilinear form. $N$-soliton solutions of the Eq. (1.3) will be obtained via Hirota bilinear method. In Sec. 3-5, based on the bilinear form of Eq. (1.3), we will construct its bilinear BT, Lax pair, Darboux covariant Lax pair and infinitely many conservation laws, respectively. In Sec. 6, based on the bilinear form of Eq. (1.2), we will construct its Riemann theta function periodic wave solution. A limiting procedure is presented to analyze in detail the asymptotic behavior of the one periodic wave and the relations between the one periodic wave 
solution and one soliton solution. Sec. 7 will contain our conclusions. Finally, some introduction of Bell polynomials and Riemann theta function are given in Appendix A and Appendix B, respectively.

\section{Bilinear representations}

In this section, we construct the bilinear representation of Eq. (1.2) and Eq. (1.3) via binary Bell polynomials approach.

Theorem 1. Under the transformation

$$
u=2(\ln F)_{2 x},
$$

the Eq. (1.2) and Eq. (1.3) can be bilinearized into

$$
\left[D_{t} D_{x}+D_{x}^{2}+\alpha D_{x}^{4}+\alpha^{2} c_{4} D_{x}^{6}-c\right] F \cdot F=0,
$$

and

$$
\begin{aligned}
& {\left[D_{x} D_{y}+D_{x}^{4}\right] F \cdot F=0,} \\
& {\left[D_{t} D_{x}+D_{x}^{2}+\alpha D_{x}^{4}+\alpha^{2} c_{4} D_{x}^{6}-\frac{5}{3} \alpha^{2} \alpha_{4}\left(D_{x}^{3} D_{y}+D_{y}^{2}\right)-c\right] F \cdot F=0,}
\end{aligned}
$$

respectively, where $c$ is an arbitrary constant.

Proof. Introducing a dimensionless field $q$ by setting

$$
u=h q_{2 x},
$$

with $h$ being free constant to be determined such that eKdV equation (1.1) linked with $P$-polynomials (A.8).

Hereby, substituting transformation (2.4) into $\mathrm{KdV}$ equation (1.1), we can write the resulting equation as follows

$$
q_{x, t}+q_{2 x}+\alpha\left(3 q_{2 x}^{2}+q_{4 x}\right)+\alpha^{2}\left(\frac{1}{3} c_{1} q_{2 x}^{3}+\frac{1}{2} c_{2} q_{3 x}^{2}+c_{3} q_{2 x} q_{4 x}-\frac{1}{2} c_{3} q_{3 x}^{2}+c_{4} q_{6 x}\right)-c=0
$$

with the choice of $h=1$ and $c$ is an arbitrary constant.

- Case 1: $c_{1}=45 c_{4}, c_{2}=c_{3}=15 c_{4}$. Eq. (2.5) can be rewritten as

$$
E(q)=q_{x, t}+q_{2 x}+\alpha\left(q_{4 x}+3 q_{2 x}^{2}\right)+\alpha^{2} c_{4}\left(15 q_{2 x}^{3}+15 q_{2 x} q_{4 x}+q_{6 x}\right)-c=0,
$$

which can be cast into a combination form of $P$-polynomials (A.8)

$$
P_{x, t}(q)+P_{2 x}(q)+\alpha P_{4 x}(q)+\alpha^{2} c_{4} P_{6 x}(q)-c P_{0 x}(q)=0 .
$$


Thus, according to the relations between $P$-polynomials and Hirota bilinear operators (A.7), bilinear representation (2.2) can be derived directly from (2.7) under the change of the dependent variable

$$
q=2 \ln F \Longleftrightarrow u=q_{2 x}=2(\ln F)_{2 x} .
$$

- Case 2: $c_{1}=30 c_{4}, c_{2}=20 c_{4}, c_{3}=10 c_{4}$. In this case, Eq. (2.5) can be rewritten as

$$
E(q)=q_{x, t}+q_{2 x}+\alpha\left(q_{4 x}+3 q_{2 x}^{2}\right)+\alpha^{2} c_{4}\left(10 q_{2 x}^{3}+5 q_{3 x}^{2}+10 q_{2 x} q_{4 x}+q_{6 x}\right)-c=0,
$$

which can be decomposed into

$$
q_{x, t}+q_{2 x}+\alpha\left(q_{4 x}+3 q_{2 x}^{2}\right)+\alpha^{2} c_{4}\left(15 q_{2 x}^{3}+15 q_{2 x} q_{4 x}+q_{6 x}\right)+\alpha^{2} c_{4}\left(5 q_{3 x}^{2}-5 q_{2 x} q_{4 x}-5 q_{2 x}^{3}\right)-c=0 .
$$

In order to write Eq. (2.10) as the combination form of $P$-polynomials, we introduce an auxiliary variable $y$ and impose a subsidiary constraint condition

$$
\left(q_{4 x}+3 q_{2 x}^{2}\right)+q_{x, y}=0
$$

on account of which, Eq. (2.10) becomes

$$
q_{x, t}+q_{2 x}+\alpha\left(q_{4 x}+3 q_{2 x}^{2}\right)+\alpha^{2} c_{4}\left(15 q_{2 x}^{3}+15 q_{2 x} q_{4 x}+q_{6 x}\right)-\frac{5}{3} \alpha^{2} c_{4}\left(q_{3 x, y}+3 q_{2 x} q_{x, y}+q_{2 y}\right)-c=0 .
$$

Thus, Eqs. (2.11) and (2.12) can be cast into a couple of combination form of $P$-polynomials

$$
\begin{aligned}
& P_{4 x}(q)+P_{x, y}(q)=0, \\
& P_{x, t}(q)+P_{2 x}(q)+\alpha P_{4 x}(q)+\alpha^{2} c_{4} P_{6 x}(q)-\frac{5}{3} \alpha^{2} c_{4}\left[P_{3 x, y}(q)+P_{2 y}(q)\right]-c P_{0 x}(q)=0 .
\end{aligned}
$$

Based on system (2.13), similar to case 1, the bilinear representation (2.3) can be directly obtained.

From the bilinear equations (2.3), $N$ soliton solutions of the Lax-type equation (1.3) can be obtained as below:

$$
\begin{aligned}
& u=2\left[\ln \left(\sum_{\mu=0,1} e^{\sum_{j=1}^{n} \mu_{j} \xi_{j}+\sum_{1 \leq j<l}^{n} \mu_{j} \mu_{l} A_{j l}}\right)\right]_{2 x}, e^{A_{j l} l}=\frac{\left(k_{j}-k_{l}\right)^{2}}{\left(k_{j}+k_{l}\right)^{2}}, j<l, j, l=1,2,3, \ldots, \\
& \xi_{j}=k_{j} x+\iota_{j} t+\zeta_{j}=k_{j} x-k_{j}\left(\alpha^{2} c_{4} k_{j}^{4}+\alpha k_{j}^{2}+1\right) t+\zeta_{j},
\end{aligned}
$$

where $\sum_{\mu=0,1}$ indicates the summation over all possible combination of $\mu_{j}=0,1(j=1,2, \ldots)$.

For example, taking $n=1$, the one soliton solution of the Lax-type equation (1.3) can be written as below:

$$
u=2\left[\ln \left(1+e^{\xi_{1}}\right)\right]_{2 x}=\frac{k^{2}}{2} \operatorname{sech}^{2}\left[\frac{k_{1}}{2}\left(x-\alpha^{2} c_{4} k_{1}^{4} t-\alpha k_{1}^{2} t-t\right)+\frac{\zeta_{1}}{2}\right] .
$$

The two soliton solution and three soliton solution can also be obtained by taking $n=2$ and $n=3$, respectively.

Figures B.1-B.3 are depicted to graphically discuss the propagation and evolution of two solitons. It can be shown that Figures B.1-B.3 lead to the following conclusions 
- The sign of the velocity $v$ can control the collisions: the collision will be head-on (Figure B.3) as we choose the opposite sign of the two soliton velocities, meanwhile the the same sign of the two soliton velocities leads to the overtaking case (Figures B.1 and B.2).

- Figure B.1 illustrates the soliton with the larger amplitude travels faster and catches up with the smaller one, while the smaller one catches up with the larger one in Figure B.2.

- Figures B.1-B.3 have in common that the two solitons maintain their original shapes and amplitudes except for phase shifts after the collision.

\section{Bilinear BT and Lax pair}

\subsection{Bilinear $B T$}

In this section, we construct the bilinear BT and the Lax pair of the Lax-type equation (1.3).

Theorem 2. Suppose that $F$ is a solution of the bilinear equation (2.3), then $G$ satisfying

$$
\begin{aligned}
& {\left[D_{x}^{2}-\lambda\right] F \cdot G=0} \\
& {\left[D_{t}+\left(15 c_{4} \alpha^{2} \lambda^{2}+3 \alpha \lambda+1\right) D_{x}+\alpha D_{x}^{3}+\alpha^{2} c_{4} D_{x}^{5}-\varrho\right] F \cdot G=0}
\end{aligned}
$$

is another solution of Lax-type equation (1.3), where $\lambda$ is spectral parameter and $\varrho$ is an arbitrary constant. Therefore, the system (3.1) is called a bilinear BT for Lax-type equation (1.3).

Proof. In order to obtain the bilinear BT of the Lax-type equation (1.3), let

$$
q=2 \ln G \text { and } q^{\prime}=2 \ln F,
$$

be two different solutions of Eq. (2.9), respectively. On introducing two new variables

$$
v=\left(q^{\prime}-q\right) / 2=\ln (F / G), w=\left(q^{\prime}+q\right) / 2=\ln (F G),
$$

we associate the two-field condition

$$
\begin{aligned}
E\left(q^{\prime}\right)-E(q) & =E(w+v)-E(w-v) \\
& =2\left[v_{x, t}+v_{2 x}+\alpha\left(v_{4 x}+6 w_{2 x} v_{2 x}\right)+\alpha^{2} c_{4}\left(10 v_{3 x} w_{3 x}+30 w_{2 x}^{2} v_{2 x}+v_{6 x}+10 v_{4 x} w_{2 x}+10 v_{2 x}^{3}\right)\right] \\
& =2 \partial_{x}\left[\mathscr{Y}_{t}(v)+\mathscr{Y}_{x}(v)+\alpha \mathscr{Y}_{3 x}(v, w)+\alpha^{2} c_{4} \mathscr{Y}_{5 x}(v, w)\right]+\mathscr{R}(v, w)=0,
\end{aligned}
$$

with

$$
\begin{aligned}
\mathscr{R}(v, w)= & \alpha\left(6 w_{2 x} v_{2 x}-6 v_{x}^{2} v_{2 x}-6 w_{3 x} v_{x}\right)+\alpha^{2} c_{4}\left(10 w_{4 x} v_{2 x}+20 v_{2 x}^{3}+30 w_{2 x}^{2} v_{2 x}-10 w_{5 x} v_{x}-20 v_{4 x} v_{x}^{2}\right. \\
& \left.-40 v_{3 x} v_{x} v_{2 x}-60 w_{2 x} v_{x} w_{3 x}-20 w_{3 x} v_{x}^{3}-60 w_{2 x} v_{x}^{2} v_{2 x}-10 v_{x}^{4} v_{2 x}\right) .
\end{aligned}
$$


Then, the next step is to decouple the two-field condition (3.4) into a pair of constraints. Thus, a auxiliary constraint should be introduced which enable ones to express $\mathscr{R}(v, w)$ as the $x$-derivative of a combination of $\mathscr{Y}$ polynomials. The simplest possible choice of such constraint may be

$$
\mathscr{Y}_{2 x}(v, w)=w_{2 x}+v_{x}^{2}-\lambda=0
$$

where $\lambda$ is an arbitrary constant called spectral parameter. In terms of the constraint (3.5), $\mathscr{R}(v, w)$ can be rewritten as

$$
R(v, w)=6 \alpha \lambda v_{2 x}+30 \alpha^{2} c_{4} \lambda^{2} v_{2 x}=2 \partial_{x}\left(3 \alpha \lambda+15 c_{4} \alpha^{2} \lambda^{2}\right) \mathscr{Y}_{x}(v)
$$

with the help of the following relations

$$
w_{2 x}=\lambda-v_{x}^{2} \text { and } w_{3 x}=-2 v_{x} v_{2 x}
$$

Then, combining the Eqs. (3.4-3.6), we deduce a coupled system of $\mathscr{Y}$-polynomials expression

$$
\begin{aligned}
& \mathscr{Y}_{2 x}(v, w)-\lambda=0, \\
& \partial_{t} \mathscr{Y}_{x}(v)+\partial_{x}\left[\left(15 c_{4} \alpha^{2} \lambda^{2}+3 \alpha \lambda+1\right) \mathscr{Y}_{x}(v)+\alpha \mathscr{Y}_{3 x}(v, w)+\alpha^{2} c_{4} \mathscr{Y}_{5 x}(v, w)-\varrho\right]=0,
\end{aligned}
$$

where the second equation can be used to construct conservation laws later. Based on the identity (A.5), the system (3.7) immediately leads to the bilinear BT (3.1).

\subsection{Lax pair}

Theorem 3. The Lax-type equation (1.3) admits the following Lax pair

$$
\begin{aligned}
& L_{1} \psi=\left(\partial_{x}^{2}+u\right) \psi=\lambda \psi \\
& \left(\psi_{t}+L_{2}\right) \psi=\left[\partial_{t}+\partial_{x}+\alpha\left(\partial_{x}^{3}+3 u \partial_{x}+3 \lambda \partial_{x}\right)+\alpha^{2} c_{4}\left(\partial_{x}^{5}+5 u_{2 x} \partial_{x}+15 u^{2} \partial_{x}+10 u \partial_{x}^{3}+15 \lambda^{2} \partial_{x}\right)-\varrho\right] \psi
\end{aligned}
$$

where $\varrho$ is an arbitrary constant and $u$ is a solution of Lax-type equation (1.3).

Proof. By transformation $v=\ln \psi$, it follows from the formulae (A.9) and (A.10) that

$$
\begin{aligned}
& \mathscr{Y}_{t}(v)=\frac{\psi_{t}}{\psi}, \mathscr{Y}_{x}(v)=\frac{\psi_{x}}{\psi}, \mathscr{Y}_{2 x}(v, w)=q_{2 x}+\frac{\psi_{2 x}}{\psi}, \mathscr{Y}_{3 x}(v, w)=3 q_{2 x} \frac{\psi_{x}}{\psi}+\frac{\psi_{3 x}}{\psi}, \\
& \mathscr{Y}_{5 x}(v, w)=\frac{\psi_{5 x}}{\psi}+10 q_{2 x} \frac{\psi_{3 x}}{\psi}+5\left(q_{4 x}+3 q_{2 x}^{2}\right) \frac{\psi_{x}}{\psi} .
\end{aligned}
$$

Then, with the help of (3.9), the system (3.7) is then linearized into a couple of equations with double parameters $\lambda$ and $\varrho$

$$
\begin{aligned}
& L_{1} \psi=\left(\partial_{x}^{2}+q_{2 x}\right) \psi=\lambda \psi \\
& \left(\psi_{t}+L_{2}\right) \psi=\left[\partial_{t}+\partial_{x}+\alpha\left(\partial_{x}^{3}+3 q_{2 x} \partial_{x}+3 \lambda \partial_{x}\right)+\alpha^{2} c_{4}\left(\partial_{x}^{5}+5 q_{4 x} \partial_{x}+15 q_{2 x}^{2} \partial_{x}+10 q_{2 x} \partial_{x}^{3}+15 \lambda^{2} \partial_{x}\right)-\varrho\right] \psi
\end{aligned}
$$


which is equivalent to the system (3.8) by replacing $q_{2 x}$ with $u$. It is easy to check that the integrability condition of

$$
\left[\partial_{t}+L_{2}, L_{1}-\lambda\right]=u_{t}+u_{x}+\alpha\left(6 u u_{x}+u_{3 x}\right)+\alpha^{2} c_{4}\left(20 u_{x} u_{2 x}+10 u u_{3 x}+30 u^{2} u_{x}+u_{5 x}\right)=0,
$$

exactly gives the Lax-type equation (1.3). Thus, system (3.8) is called the Lax pair of Lax-type equation (1.3).

\section{Darboux covariant Lax pair}

In this section, we construct a kind of Darboux covariant Lax pair whose form is invariant under a certain gauge transformation.

Theorem 4. Using the associated Lax pair (3.8), the Lax-type equation (1.3) admits a kind of Darboux covariant Lax pair as follows:

$$
\begin{gathered}
\tilde{L}_{1} \psi=\left(\partial_{x}^{2}+\tilde{q}_{2 x}\right) \psi=\lambda \psi, \\
\left(\psi_{t}+\tilde{L}_{2, \operatorname{cov}}\right) \psi=\left[\partial_{x}+\partial_{t}+\alpha\left(4 \partial_{x}^{3}+3 \tilde{q}_{3 x}+6 \tilde{q}_{2 x} \partial_{x}\right)+\alpha^{2} c_{4}\left(40 \tilde{q}_{2 x} \partial_{x}^{3}+60 \tilde{q}_{3 x} \partial_{x}^{2}+50 \tilde{q}_{4 x} \partial_{x}+16 \partial_{x}^{5}+\right.\right. \\
\left.\left.15 \tilde{q}_{5 x}+30 \tilde{q}_{2 x} \tilde{q}_{3 x}+30 \tilde{q}_{2 x}^{2} \partial_{x}\right)-\varrho\right] \psi,
\end{gathered}
$$

whose form is Darboux covariant, namely

$$
\begin{aligned}
& T L_{1}(q) T^{-1}=\tilde{L}_{1}(\tilde{q}), \\
& T\left(\partial_{t}+L_{2, \operatorname{cov}}\right)(q) T^{-1}=\left(\partial_{t}+\tilde{L}_{2, \operatorname{cov}}\right)(\tilde{q}),
\end{aligned}
$$

with $\tilde{q}=q+2 \ln \psi$, under a certain gauge transformation

$$
T=\psi \partial_{x} \psi^{-1}=\partial_{x}-\sigma, \sigma=\partial_{x} \ln \psi
$$

Proof. To begin with, we let $\psi$ is a solution of the first equation of Lax pair (3.10). It can be verified that the gauge transformation (4.3) maps the operator $L_{1}(q)-\lambda$ onto a similar operator

$$
T\left(L_{1}(q)-\lambda\right) T^{-1}=\tilde{L}_{1}(\tilde{q})-\lambda
$$

which satisfies the covariance condition

$$
\tilde{L}_{1}(\tilde{q})=L_{1}(\tilde{q}=q+\Delta q) \text { with } \Delta q=2 \ln \psi
$$

But it can be verified that similar property does not hold for the second evolution equation of Lax pair (3.10). However, one can find another third order operator $L_{2, \operatorname{cov}}(q)$ with appropriate coefficients, such that $\partial_{t}+L_{2, \operatorname{cov}}(q)$ be mapped, by gauge transformation (4.3), onto a similar operator $\tilde{L}_{2, \operatorname{cov}}(\tilde{q})$ which satisfies the covariance condition

$$
\tilde{L}_{2, \operatorname{cov}}(\tilde{q})=L_{2, \operatorname{cov}}(\tilde{q}=q+\Delta q)
$$


Therefore, assuming that $\psi$ is a solution of the following Lax pair

$$
L_{1} \psi=\lambda \psi,\left(\partial_{t}+L_{2, \operatorname{cov}}\right) \psi=0
$$

with

$$
L_{1}=\partial_{x}^{2}+q_{2 x}, L_{2, \mathrm{cov}}=16 \alpha^{2} c_{4}\left(\partial_{x}^{5}+b_{1} \partial_{x}^{3}+b_{2} \partial_{x}^{2}+b_{3} \partial_{x}+b_{4}\right)+4 \alpha\left(\partial_{x}^{3}+b_{5} \partial_{x}+b_{6}\right)+\partial_{x},
$$

and $b_{1}, \ldots, b_{6}$ are functions to be determined. It suffices that we require the transformation (4.3) map the operator $\partial_{t}+L_{2, \operatorname{cov}}$ onto the similar one

$$
T\left(\partial_{t}+L_{2, \mathrm{cov}}\right) T^{-1}=\partial_{t}+\tilde{L}_{2, \mathrm{cov}},
$$

where

$$
\tilde{L}_{2, \operatorname{cov}}=\partial_{t}+16 \alpha^{2} c_{4}\left(\partial_{x}^{5}+\tilde{b}_{1} \partial_{x}^{3}+\tilde{b}_{2} \partial_{x}^{2}+\tilde{b}_{3} \partial_{x}+\tilde{b}_{4}\right)+4 \alpha\left(\partial_{x}^{3}+\tilde{b}_{5} \partial_{x}+\tilde{b}_{6}\right)+\partial_{x},
$$

with $\tilde{b}_{1}, \ldots, \tilde{b}_{6}$ satisfy the following covariant condition

$$
\tilde{b}_{j}=b_{j}(q)+\Delta b_{j}=b_{j}(q+\Delta q), j=1, \ldots, 6 .
$$

By virtue of (4.7) and (4.8), we can find that

$$
\begin{aligned}
& \Delta b_{1}=\tilde{b}_{1}-b_{1}=5 \sigma_{x}, \\
& \Delta b_{2}=\tilde{b}_{2}-b_{2}=b_{1, x}+10 \sigma_{2 x}+\Delta b_{1} \sigma, \\
& \Delta b_{3}=\tilde{b}_{3}-b_{3}=\sigma \Delta \tilde{b}_{2}+3 \sigma_{x} \tilde{b}_{1}+b_{3}+10 \sigma_{3 x}+b_{2, x}, \\
& \Delta b_{4}=\tilde{b}_{4}-b_{4}=5 \sigma_{4 x}+b_{3, x}+b_{4}+3 \sigma_{2 x} \tilde{b}_{1}+2 \sigma_{x} \tilde{b}_{2}+\sigma \Delta b_{3}, \\
& \Delta b_{5}=\tilde{b}_{5}-b_{5}=3 \sigma_{x}, \\
& \Delta b_{6}=\tilde{b}_{6}-b_{6}=\tilde{b}_{5, x}+b_{6}+3 \sigma \sigma_{x},
\end{aligned}
$$

and

$$
\sigma_{t}+\sigma_{x}+4 \alpha\left(\Delta b_{6} \sigma+\sigma_{3 x}+b_{6, x}+\tilde{b}_{5} \sigma_{x}\right)+16 \alpha^{2} c_{4}\left(\sigma_{5 x}+\tilde{b}_{3} \sigma_{x}+b_{4, x}+\tilde{b}_{4} \sigma+\tilde{b}_{1} \sigma_{3 x}-b_{4} \sigma+\tilde{b}_{2} \sigma_{2 x}\right)=0 .
$$

In terms of relation (4.10), it remains to determine $b_{1}, \ldots, b_{6}$ in the form of polynomial expressions in terms of derivatives of $q$

$$
b_{j}=F_{j}\left(q, q_{x}, q_{2 x}, q_{3 x}, \ldots\right), j=1, \ldots, 6,
$$

such that

$$
\Delta F_{j}=F_{j}\left(q+\Delta q, q_{x}+\Delta q_{x}, \ldots\right)-F_{j}\left(q, q_{x}, \ldots\right)=\Delta b_{j},
$$


with $\Delta q_{k x}=2(\ln q)_{k x}, k=1, \ldots, 6$ and the $\Delta b_{j}$ being determined by the relations (4.11).

Thus, in order to satisfy the first condition

$$
\Delta b_{1}=\Delta F_{1}=F_{1, q} \Delta q+F_{1, q_{x}} \Delta q_{x}+\ldots=5 \sigma_{x}=\frac{5}{2} \Delta q_{2 x},
$$

one chooses

$$
b_{1}=F_{1}\left(q_{2 x}\right)=\frac{5}{2} q_{2 x}+d_{1},
$$

with $d_{1}$ being arbitrary constant.

Duing to the relation (4.11b) contains the term $b_{1, x}=\frac{5}{2} q_{3 x}$, which should be eliminated such that $\Delta b_{2}$ admits the form (4.14). It follows from the eigenvalue equation in (4.7), we have

$$
q_{3 x}=-\sigma_{2 x}-2 \sigma \sigma_{x} .
$$

Substituting (4.16) and (4.17) into (4.11b) yields

$$
\Delta b_{2}=\frac{15}{2} \sigma_{2 x}=\frac{15}{4} \Delta q_{3 x} .
$$

It is can be verified that the second condition is satisfied

$$
\Delta F_{2}=F_{2, q} \Delta q+F_{2, q_{x}} \Delta q_{x}+\ldots=\Delta b_{2},
$$

if one chooses

$$
b_{2}=F_{2}\left(q_{3 x}\right)=\frac{15}{4} q_{3 x}+d_{2},
$$

in which $d_{2}$ being arbitrary constant.

Proceeding in the same way, we have

$$
\begin{aligned}
& b_{3}=\frac{25}{8} q_{4 x}+\frac{15}{8} q_{2 x}^{2}+d_{3}, \\
& b_{4}=\frac{15}{16} q_{5 x}+\frac{15}{8} q_{2 x} q_{3 x}+d_{4}, \\
& b_{5}=\frac{3}{2} q_{2 x}+d_{5}, \\
& b_{6}=\frac{3}{4} q_{3 x}+d_{6},
\end{aligned}
$$

where $d_{3}, d_{4}, d_{5}$ and $d_{6}$ are all arbitrary constants.

Setting $d_{i}=0(i=1, \cdots, 6)$ in (4.16), (4.20) and (4.21), it follows from (4.7) that we find the following Darboux covariant evolution equation

$$
\begin{aligned}
& \psi_{t}+L_{2, \operatorname{cov} \psi}=0, \\
& \begin{aligned}
L_{2, \operatorname{cov}}= & \partial_{x}+\alpha\left(4 \partial_{x}^{3}+3 q_{3 x}+6 q_{2 x} \partial_{x}\right)+\alpha^{2} c_{4}\left(40 q_{2 x} \partial_{x}^{3}+60 q_{3 x} \partial_{x}^{2}+50 q_{4 x} \partial_{x}+16 \partial_{x}^{5}+15 q_{5 x}\right. \\
& \left.+30 q_{2 x} q_{3 x}+30 q_{2 x}^{2} \partial_{x}\right),
\end{aligned}
\end{aligned}
$$


which is in agreement with Eq. (4.12).

The integrability condition of the Darboux covariant Lax pair (4.7) precisely gives rise to Lax-type equation (1.3) in Lax representation

$$
\left[\partial_{t}+L_{2, \operatorname{cov}}, L_{1}-\lambda\right]=u_{t}+u_{x}+\alpha\left(6 u u_{x}+u_{3 x}\right)+\alpha^{2} c_{4}\left(20 u_{x} u_{2 x}+10 u u_{3 x}+30 u^{2} u_{x}+u_{5 x}\right)=0,
$$

which implies that system (4.7) are also a Lax pair for the Lax-type equation (1.3).

Moreover, the relation between the operator $L_{2, \mathrm{cov}}$ and the operator $L_{2}$ is given by

$$
L_{2, \operatorname{cov}}=L_{2}+3 \alpha \partial_{x}\left(L_{1}-\lambda\right)+\alpha^{2} c_{4}\left[15\left(\partial_{x}^{3}+\lambda \partial_{x}+u \partial_{x}+u_{x}\right)\left(L_{1}-\lambda\right)+\varrho\right] .
$$

It is note that the higher-order members of Lax-type equation (1.3) can be obtained in a similar way step by step.

\section{Infinitely many conservation laws}

In this section, we derive the infinitely many conservation laws for the Lax-type equation (1.3) by using the binary Bell polynomials.

Theorem 5. The Lax-type equation (1.3) admits an infinitely many conservation laws

$$
\mathscr{I}_{n, t}+\mathscr{F}_{n, x}=0, n=1,2, \ldots
$$

The conversed densities $\mathscr{I}_{n}^{\prime}$ s are given by the recursion formulas

$$
\begin{aligned}
& \mathscr{I}_{1}=-\frac{1}{2} q_{2 x}=-\frac{1}{2} u, \\
& \mathscr{I}_{2}=-\frac{1}{2} \mathscr{I}_{1, x}=\frac{1}{4} q_{3 x}=\frac{1}{4} u_{x}, \\
& \ldots, \\
& \mathscr{I}_{n+1}=-\frac{1}{2}\left(\mathscr{I}_{n, x}+\sum_{k=1}^{n} \mathscr{I}_{k} \mathscr{I}_{n-k}\right), n=2,3, \ldots,
\end{aligned}
$$

and the fluxes $\mathscr{F}_{n}^{\prime}$ s are given by

$$
\begin{aligned}
\mathscr{F}_{1}= & -\frac{u}{2}-\frac{1}{2} \alpha\left(3 u^{2}+u_{2 x}\right)-\alpha^{2} c_{4}\left(5 u^{3}+\frac{5}{2} u_{x}^{2}+\frac{1}{2} u_{4 x}+5 u u_{2 x}\right), \\
\mathscr{F}_{2}= & \frac{1}{4} u_{x}+\alpha\left(\frac{1}{4} u_{3 x}+\frac{3}{2} u u_{x}\right)+\alpha^{2} c_{4}\left(5 u_{x} u_{2 x}+\frac{5}{2} u u_{3 x}+\frac{1}{4} u_{5 x}+\frac{15}{2} u^{2} u_{x}\right), \\
\ldots, & \\
& \quad 10 \sum_{i+j+k=n}\left(\mathscr{I}_{i} \mathscr{I}_{j} \mathscr{I}_{k, 2 x}+\mathscr{I}_{i} \mathscr{I}_{j, x} \mathscr{I}_{k, x}\right)-10 \sum_{i+j=n+1}\left(2 \mathscr{I}_{i} \mathscr{I}_{j, 2 x}+\mathscr{I}_{i, x} \mathscr{I}_{j, x}\right)+30 \sum_{i+j+k+k+l=n} \mathscr{I}_{i} \mathscr{I}_{j} \mathscr{I}_{k} \mathscr{I}_{l} \\
& \left.+6 \sum_{i+j+k+l+m=n} \mathscr{I}_{i} \mathscr{I}_{j} \mathscr{I}_{j} \mathscr{I}_{k} \mathscr{I}_{l} \mathscr{I}_{m}\right) .
\end{aligned}
$$


Proof. The conservation laws actually have been hinted in the two-filed constraint system (3.7), which can be rewritten in the conserved form

$$
\begin{aligned}
& \mathscr{Y}_{2 x}(v, w)-\lambda=0, \\
& \partial_{t} \mathscr{Y}_{x}(v)+\partial_{x}\left[\left(15 c_{4} \alpha^{2} \lambda^{2}+3 \alpha \lambda+1\right) \mathscr{Y}_{x}(v)+\alpha \mathscr{Y}_{3 x}(v, w)+\alpha^{2} c_{4} \mathscr{Y}_{5 x}(v, w)\right]=0,
\end{aligned}
$$

by using the relation

$$
\partial_{t} \mathscr{Y}_{x}(v)=\partial_{x} \mathscr{Y}_{t}(v)=v_{x, t},
$$

with $\varrho=0$.

By introducing a new potential function

$$
\eta=\frac{q_{x}^{\prime}-q_{x}}{2}
$$

it follows from the relation (3.3) that

$$
v_{x}=\eta, w_{x}=q_{x}+\eta .
$$

Substituting (5.6) into (5.4), we get a Riccati-type equation

$$
\eta_{x}+\eta^{2}+q_{2 x}-\varepsilon^{2}=0
$$

and a divergence-type equation

$$
\begin{aligned}
& \eta_{t}+\left[30 \alpha^{2} c_{4} \eta \varepsilon^{4}-2 \alpha\left(10 \alpha c_{4} \eta^{3}-5 \alpha c_{4} \eta_{2 x}-3 \eta\right) \varepsilon^{2}-10 \alpha^{2} c_{4} \eta^{2} \eta_{2 x}-10 \alpha^{2} c_{4} \eta \eta_{x}^{2}+\eta+6 \alpha^{2} c_{4} \eta^{5}+\alpha \eta_{2 x}\right. \\
& \left.+\alpha^{2} c_{4} \eta_{4 x}-2 \alpha \eta^{3}\right]_{x}=0
\end{aligned}
$$

with $\lambda=\varepsilon^{2}$.

To proceed, inserting the expansion

$$
\eta=\varepsilon+\sum_{n=1}^{\infty} \mathscr{I}_{n}\left(q, q_{x}, \ldots\right) \varepsilon^{-n},
$$

into (5.7) and equating the coefficients for power of $\varepsilon$, we explicitly obtain the recursion relations (5.2) for the conserved densities $\mathscr{I}_{n}^{\prime} s$.

Furthermore, substituting expansion (5.9) into the divergence-type equation (5.8) leads to

$$
\begin{aligned}
& \sum_{n=1}^{\infty} \mathscr{I}_{n, t} \varepsilon^{-n}+\left\{30 \alpha^{2} c_{4} \varepsilon^{4}\left(\varepsilon+\sum_{n=1}^{\infty} \mathscr{I}_{n} \varepsilon^{-n}\right)-2 \alpha\left[10 \alpha c_{4}\left(\varepsilon+\sum_{n=1}^{\infty} \mathscr{I}_{n} \varepsilon^{-n}\right)^{3}-5 \alpha c_{4} \sum_{n=1}^{\infty} \mathscr{I}_{n, 2 x} \varepsilon^{-n}-3\left(\varepsilon+\sum_{n=1}^{\infty} \mathscr{I}_{n} \varepsilon^{-n}\right)\right] \varepsilon^{2}\right. \\
& -10 \alpha^{2} c_{4}\left(\varepsilon+\sum_{n=1}^{\infty} \mathscr{I}_{n} \varepsilon^{-n}\right)^{2} \sum_{n=1}^{\infty} \mathscr{I}_{n, 2 x} \varepsilon^{-n}-10 \alpha^{2} c_{4}\left(\varepsilon+\sum_{n=1}^{\infty} \mathscr{I}_{n} \varepsilon^{-n}\right)\left(\sum_{n=1}^{\infty} \mathscr{I}_{n, x} \varepsilon^{-n}\right)^{2}+\varepsilon+\sum_{n=1}^{\infty} \mathscr{I}_{n} \varepsilon^{-n} \\
& \left.+6 \alpha^{2} c_{4}\left(\varepsilon+\sum_{n=1}^{\infty} \mathscr{I}_{n} \varepsilon^{-n}\right)^{5}+\alpha \sum_{n=1}^{\infty} \mathscr{I}_{n, 2 x} \varepsilon^{-n}+\alpha^{2} c_{4} \sum_{n=1}^{\infty} \mathscr{I}_{n, 4 x} \varepsilon^{-n}-2 \alpha\left(\varepsilon+\sum_{n=1}^{\infty} \mathscr{I}_{n} \varepsilon^{-n}\right)^{3}\right\}_{x}=0
\end{aligned}
$$


which provides us the infinitely many conservation laws (5.1).

In equation (5.1), the conversed densities $\mathscr{I}_{n}^{\prime} s$ are given by recursion formulas (5.2), and the fluxes $\mathscr{F}_{n}^{\prime} s$ are obtained by (5.3) through a cumbersome calculation. The first equation of conservation law (5.1) is exactly the Laxtype equation (1.3).

\section{Quasi-periodic wave solution and asymptotic property}

A. Nakamura [20, 21] proposed a convenient way to construct a kind of quasi-periodic solutions of nonlinear equations by means of the Hirota bilinear method and Riemann theta function. More recently, this method is extended to investigate many NLEEs which inclue both continuous and discrete ones [22-27]. In the following, we construct the one periodic wave solution of Sawada-Kotera-type equation (1.2) by this method and discuss its asymptotic property in detail.

\subsection{Quasi-periodic wave solution}

Based on the bilinear form (2.2) of Sawada-Kotera-type equation (1.2)

$$
\left[D_{t} D_{x}+D_{x}^{2}+\alpha D_{x}^{4}+\alpha^{2} c_{4} D_{x}^{6}-c\right] F \cdot F=0,
$$

with $c$ is a nonzero constant, we consider Riemann theta function solution of Sawada-Kotera-type equation (1.2)

$$
\vartheta(\theta)=\vartheta(\theta, \tau)=\sum_{n \in Z^{N}} e^{\pi i\langle n \tau, n\rangle+2 \pi i\langle\theta, n\rangle}
$$

where the integer value vector $n=\left(n_{1}, n_{2}, \ldots, n_{N}\right)^{T} \in Z^{N}$, the complex phase variables $\theta=\left(\theta_{1}, \theta_{2}, \ldots, \theta_{N}\right)^{T} \in Z^{N}$ and $-i \tau$ is a positive definite and real-valued symmetric $N \times N$ matrix.

Theorem 6. Assuming that $\vartheta(\theta, \tau)$ is a Riemann theta function for $N=1$ with $\theta=\rho_{j} x+\omega_{j} t+\gamma_{j}, j=0,1,2, \ldots$, the Sawada-Kotera-type equation (1.2) admits a one periodic wave solution as follows:

$$
u=2 \partial_{x}^{2} \ln \vartheta(\theta, \tau)
$$

where

$$
\omega=\frac{a_{12} b_{2}-b_{1} a_{22}}{a_{11} a_{22}-a_{21} a_{12}}, c=\frac{a_{11} b_{2}-b_{1} a_{21}}{a_{11} a_{22}-a_{21} a_{12}},
$$


with

$$
\begin{aligned}
& \delta=e^{\pi i \tau}, a_{11}=\sum_{n=-\infty}^{+\infty}-16 n^{2} \pi^{2} \rho \delta^{2 n^{2}}, a_{12}=\sum_{n=-\infty}^{+\infty} \delta^{2 n^{2}}, \\
& b_{1}=\sum_{n=-\infty}^{+\infty}\left(-16 n^{2} \pi^{2} \rho^{2}+256 \alpha n^{4} \pi^{4} \rho^{4}-4096 \alpha^{2} c_{4} n^{6} \pi^{6} \rho^{6}\right) \delta^{2 n^{2}}, \\
& a_{21}=\sum_{n=-\infty}^{+\infty}\left[-4(2 n-1)^{2} \pi^{2} \rho\right] \delta^{2 n^{2}-2 n+1}, a_{22}=\sum_{n=-\infty}^{+\infty} \delta^{2 n^{2}-2 n+1}, \\
& b_{2}=\sum_{n=-\infty}^{+\infty}\left[-4(2 n-1)^{2} \pi^{2} \rho^{2}+16 \alpha(2 n-1)^{4} \pi^{4} \rho^{4}-64 \alpha^{2} c_{4}(2 n-1)^{6} \pi^{6} \rho^{6}\right] \delta^{2 n^{2}-2 n+1},
\end{aligned}
$$

and the other parameters $\rho$ and $\gamma$ are free.

Proof. In order to obtain the one periodic wave solution of Sawada-Kotera-type equation (1.2), we consider the simplest case of the Riemann theta function $\vartheta(\theta, \tau)$ with $N=1$, namely

$$
\vartheta(\theta, \tau)=\sum_{n=-\infty}^{+\infty} e^{\pi i n^{2} \tau+2 \pi i n \theta},
$$

where the phase variable $\theta=\rho x+\omega t+\gamma$ and the parameter $\operatorname{Im} \tau>0$. Thus, $\rho, \omega$ and $\gamma$ satisfy the following system:

$$
\begin{aligned}
& \sum_{n=-\infty}^{+\infty} \mathscr{L}(4 n \pi i \rho, 4 n \pi i \omega) e^{2 n^{2} \pi i \tau}=0, \\
& \sum_{n=-\infty}^{+\infty} \mathscr{L}[2 \pi i(2 n-1) \rho, 2 \pi i(2 n-1) \omega] e^{\left(2 n^{2}-2 n+1\right) \pi i \tau}=0 .
\end{aligned}
$$

Substituting the bilinear form (6.1) into the system (6.7) yields

$$
\begin{aligned}
& \sum_{n=-\infty}^{+\infty}\left(-16 n^{2} \pi^{2} \rho \omega-16 n^{2} \pi^{2} \rho^{2}+256 \alpha n^{4} \pi^{4} \rho^{4}-4096 \alpha^{2} c_{4} n^{6} \pi^{6} \rho^{6}-c\right) e^{2 n^{2} \pi i \tau}=0, \\
& \sum_{n=-\infty}^{+\infty}\left[-4(2 n-1)^{2} \pi^{2} \rho \omega-4(2 n-1)^{2} \pi^{2} \rho^{2}+16 \alpha(2 n-1)^{4} \pi^{4} \rho^{4}-64 \alpha^{2} c_{4}(2 n-1)^{6} \pi^{6} \rho^{6}\right. \\
& -c] e^{\left(2 n^{2}-2 n+1\right) \pi i \tau}=0 .
\end{aligned}
$$

We introduce the notations by system (6.5), then system (6.8) is simplified into a linear system for the frequency $\omega$ and the integration constant $c$, namely

$$
\left(\begin{array}{ll}
a_{11} & a_{12} \\
a_{21} & a_{22}
\end{array}\right)\left(\begin{array}{c}
\omega \\
-c
\end{array}\right)=\left(\begin{array}{c}
-b_{1} \\
-b_{2}
\end{array}\right) .
$$

Now we obtain a one quasi-periodic wave solution of Sawada-Kotera-type equation (1.2)

$$
u=2 \partial_{x}^{2} \ln \vartheta(\theta, \tau)
$$

which provided the vector $(\omega,-c)^{T}$ solves Eq. (6.9) with the theta function $\vartheta(\theta, \tau)$ given by equation (6.6) and parameters $\omega, c$ by Eq. (6.9). The other parameters $\rho$ and $\gamma$ are free. Figure B.4 shows the propagation of the one periodic wave via solution (6.10). 


\subsection{Asymptotic property of one periodic wave solution}

Based on the bilinear representation (2.2), the one soliton solution of Sawada-Kotera-type equation (1.2) can be obtained as

$$
u=2\left[\ln \left(1+e^{\eta}\right)\right]_{2 x} \text {, with } \eta=\mu x+v t+\varsigma=\mu x-\mu\left(\alpha^{2} c_{4} \mu^{4}+\alpha \mu^{2}+1\right) t+\varsigma,
$$

on account of which, the relation between the one periodic wave solution and the one soliton solution can be directly established as follows.

Theorem 7. If the vector $(\omega,-c)^{T}$ is a solution of the system (6.9) for the one periodic wave solution, we let

$$
\rho=\frac{\mu}{2 \pi i}, \gamma=\frac{\varsigma-\pi i \tau}{2 \pi i}
$$

where $\mu$ and $s$ are given in (6.11). Then we have the following asymptotic properties:

$$
c \rightarrow 0,2 \pi i \theta \rightarrow \eta-\pi i \tau, \vartheta(\theta, \tau) \rightarrow 1+e^{\eta} \text {, as } \delta \rightarrow 0 .
$$

Proof. Based on the system (6.5), we write functions $a_{i j}, b_{i}, i, j=1,2$ as the series about $\delta$,

$$
\begin{aligned}
& a_{11}=-32 \pi^{2} \rho \delta^{2}\left(1+4 \delta^{6}+\cdots+n^{6} \delta^{2 n^{2}-2}+\cdots\right), \\
& a_{12}=1+2\left(\delta^{2}+\delta^{8}+\cdots+\delta^{2 n^{2}}\right), \\
& b_{1}=-32 \pi^{2} \rho^{2} \delta^{2}\left[\left(1-16 \alpha \pi^{2} \rho^{2}+256 \alpha^{2} c_{4} \pi^{4} \rho^{4}\right)+\delta^{6}\left(4-256 \alpha \pi^{2} \rho^{2}+16384 \alpha^{2} c_{4} \pi^{4} \rho^{4}\right)\right. \\
&\left.+\cdots+\delta^{2 n^{2}-2}\left(n^{2}-16 n^{4}+256 n^{6}\right)+\cdots\right], \\
& a_{21}=-4 \pi^{2} \rho \delta\left[2+18 \delta^{4}+98 \delta^{24}+\cdots+2(2 n-1)^{2} \delta^{2 n^{2}-2 n}+\cdots\right], \\
& a_{22}=2\left(\delta+\delta^{5}+\delta^{13}+\cdots+\delta^{2 n^{2}-2 n+1}+\cdots\right), \\
& b_{2}=-4 \pi^{2} \rho^{2} \delta\left\{\left(2-8 \alpha \pi^{2} \rho^{2}+32 \alpha^{2} c_{4} \pi^{4} \rho^{4}\right)+\delta^{4}\left(18-648 \alpha \pi^{2} \rho^{2}+23328 \alpha^{2} c_{4} \pi^{4} \rho^{4}\right)+\cdots\right. \\
&\left.+\delta^{2 n^{2}-2 n}\left[2(2 n-1)^{2}-8(2 n-1)^{4} \alpha \pi^{2} \rho^{2}+32(2 n-1)^{6} \alpha^{2} c_{4} \pi^{6} \rho^{6}\right]+\cdots\right\} .
\end{aligned}
$$

With the help of Theorem 9 in Appendix Appendix B, we have

$$
\begin{aligned}
& A_{0}=\left(\begin{array}{ll}
0 & 1 \\
0 & 0
\end{array}\right), A_{1}=\left(\begin{array}{cc}
0 & 0 \\
-8 \pi^{2} \rho & 2
\end{array}\right), A_{2}=\left(\begin{array}{cc}
-32 \pi^{2} \rho & 2 \\
0 & 0
\end{array}\right), A_{5}=\left(\begin{array}{cc}
0 & 0 \\
-72 \pi^{2} \rho & 2
\end{array}\right), \\
& A_{3}=A_{4}=0, \ldots,
\end{aligned}
$$

and

$$
\begin{aligned}
& B_{1}=\left(\begin{array}{c}
0 \\
4 \pi^{2} \rho^{2} \Delta_{1}
\end{array}\right), B_{2}=\left(\begin{array}{c}
32 \pi^{2} \rho^{2} \Delta_{2} \\
0
\end{array}\right), B_{5}=\left(\begin{array}{c}
0 \\
4 \pi^{2} \rho^{2} \Delta_{3}
\end{array}\right), \\
& B_{0}=B_{3}=B_{4}=0, \ldots,
\end{aligned}
$$


where

$$
\begin{aligned}
& \Delta_{1}=2-8 \alpha \pi^{2} \rho^{2}+32 \alpha^{2} c_{4} \pi^{4} \rho^{4}, \\
& \Delta_{2}=1-16 \alpha \pi^{2} \rho^{2}+256 \alpha^{2} c_{4} \pi^{4} \rho^{4}, \\
& \Delta_{3}=18-648 \alpha \pi^{2} \rho^{2}+23328 \alpha^{2} c_{4} \pi^{4} \rho^{4} .
\end{aligned}
$$

Substituting the system (6.15), (6.16) and (6.17) into formulas (B.8), one can obtain

$$
\begin{aligned}
& X_{0}=\left(\begin{array}{c}
-\frac{\rho}{2} \Delta_{1} \\
0
\end{array}\right), X_{2}=\left(\begin{array}{c}
-4 \rho \Delta_{1} \\
-16 \pi^{2} \rho^{2} \Delta_{1}
\end{array}\right), X_{4}=\left(\begin{array}{c}
-\frac{\rho}{2}\left(\Delta_{3}+39 \Delta_{1}\right) \\
-96 \pi^{2} \rho^{2} \Delta_{1}
\end{array}\right), \\
& X_{1}=X_{3}=0, \ldots
\end{aligned}
$$

From (B.4), one then has

$$
\begin{aligned}
& \omega=-\frac{\rho}{2} \Delta_{1}-4 \rho \Delta_{1} \delta^{2}-\frac{\rho}{2}\left(\Delta_{3}+39 \Delta_{1}\right) \delta^{4}+o\left(\delta^{4}\right), \\
& c=16 \pi^{2} \rho^{2} \Delta_{1} \delta^{2}+96 \pi^{2} \rho^{2} \Delta_{1} \delta^{4}+o\left(\delta^{4}\right)
\end{aligned}
$$

which implies by using relation (6.12) that

$$
c \rightarrow 0,
$$

$$
2 \pi i \omega \rightarrow-\pi i \rho\left(2-8 \alpha \pi^{2} \rho^{2}+32 \alpha^{2} c_{4} \pi^{4} \rho^{4}\right)=-\mu\left(1+\alpha \mu^{2}+\alpha^{2} c_{4} \mu^{4}\right), \text { when } \delta \rightarrow 0 .
$$

In order to show that the one periodic wave degenerates to the one soliton solution under the limit $\delta \rightarrow 0$, we first expand the periodic function in the form of

$$
\vartheta(\theta, \tau)=1+\left(e^{2 \pi i \theta}+e^{-2 \pi i \theta}\right) \delta+\left(e^{4 \pi i \theta}+e^{-4 \pi i \theta}\right) \delta^{4}+\ldots
$$

Using the transformation, one has

$$
\begin{aligned}
& \vartheta(\theta, \tau)=1+e^{\bar{\theta}}+\left(e^{-\bar{\theta}}+e^{2 \bar{\theta}}\right) \delta^{2}+\left(e^{-2 \bar{\theta}}+e^{3 \bar{\theta}}\right) \delta^{6}+\cdots \rightarrow 1+e^{\bar{\theta}}, \text { as } \delta \rightarrow 0, \\
& \bar{\theta}=2 \pi i \theta+\pi i \tau=\mu x+2 \pi i \omega t+\varsigma
\end{aligned}
$$

Combining equations (6.20) and (6.22), one deduces that

$$
\begin{aligned}
& \bar{\theta}=2 \pi i \theta+\pi i \tau=\mu x+2 \pi i \omega t+\varsigma=\mu x-\mu\left(1+\alpha \mu^{2}+\alpha^{2} c_{4} \mu^{4}\right)+\varsigma, \text { as } \delta \rightarrow 0, \\
& 2 \pi i \theta \rightarrow \eta-\pi i \tau, \text { as } \delta \rightarrow 0 .
\end{aligned}
$$

With the aid of equations (6.22) and (6.23), one can obtain

$$
\vartheta(\theta) \rightarrow 1+e^{\eta}, \text { as } \delta \rightarrow 0
$$




\section{Conclusions}

In present paper, with the help of the binary Bell polynomials, Hirota bilinear method and symbolic computation, we systematically investigate the integrability of Eq. (1.3) and Eq. (1.2) in fluids with the second-order nonlinear and dispersive terms.

- For Sawada-Kotera-type equation (1.2), P-polynomials expression (2.7) and bilinear form (2.2) are obtained. Employing the Hirota bilinear method, Riemann theta function and symbolic computation, we derive the one periodic wave solution (6.10) and given the corresponding Figure B.4. The exact relations between the one periodic wave solution and the one soliton solution are established. It is rigorously shown that the one periodic wave solution tend to the one soliton solution under a small amplitude limit $\delta \rightarrow 0$.

- For Lax-type equation (1.3), by introducing an an auxiliary variable $y$ and impose a subsidiary constraint condition (2.11), $P$-polynomials expression (2.13) and bilinear form (2.3) are obtained. Based on bilinear form (2.3), by virtue of the Hirota bilinear method, the $N$ soliton solution (2.14) is obtained.

- From the expression (2.9) and by choosing a suitable constraint condition (3.5), the $\mathscr{Y}$-polynomials-type BT (3.7) and bilinear BT (3.1) are obtained. With the help of formulae (A.9) and (A.10), the Lax pair (3.8) is obtained, which can also be regarded as the compatibility condition for the bilinear BT (3.1). Moreover, by appling the properties of elementary Darboux transformation, namely gauge transformation (4.3), a type of Darboux covariant Lax pair (4.1) is obtained. Note that the Darboux covariant Lax pair (4.1) can be used to find the higher-order members of Lax-type equation (1.3). Finally, a Riccati-type equation (5.7) and a divergencetype equation (5.8) are used to construct the infinitely many conservation laws for the Lax-type equation (1.3). All conserved densities (5.2) and fluxes (5.3) are given with explicit recursion formulas.

In addition, the present results in this paper demonstrate that the Bell polynomials play an important role in the characterization of bilinear BTs, Lax pairs and infinitely many conservation laws. We also believe that there are still many deep relations between generalized Bell polynomials and integrable structures, which still remain open and worth to be considered. For instance, the relations between the Bell polynomials with symmetries, Sato theory, Hamiltonian functions, etc.

\section{Acknowledgments}

This work is supported by the NNSF (Nos. 11275072 and 11075055), RFDP (No. 20120076110024), Innovative Research Team Program of the National Natural Science Foundation of China (No. 61021004), Shanghai Leading Academic Discipline Project (No. B412), National High Technology Research and Development Program (No. 2011AA010101) and Shanghai Knowledge Service Platform for Trustworthy Internet of Things (No. ZF1213). 


\section{Appendix A. Bell polynomials}

In this section, we simply recall some necessary notations on the Bell polynomials (see F. Lambert and his coworkers' work for details [7-9]).

With the assumption that $f=f(x)$ is a $\mathbb{C}^{\infty}$ function of $x$ and $f_{r x}=\partial_{x}^{r} f, r=1,2, \ldots, n$, then

$$
Y_{n x}(f) \equiv Y_{n}\left(f_{x}, \ldots, f_{n x}\right)=Y_{n}\left(\left\{f_{r x}(1 \leq n)\right\}\right)=e^{-f} \partial_{x}^{n} e^{f}, \quad f_{0 x} \equiv f
$$

i.e.

$$
Y_{x}(f)=f_{x}, Y_{2 x}(f)=f_{2 x}+f_{x}^{2}, Y_{3 x}(f)=f_{3 x}+3 f_{x} f_{2 x}+f_{x}^{3}, \ldots,
$$

is a polynomial in the derivatives of $f$ with respect to $x$, which called the one dimensional Bell polynomials or $\mathscr{Y}$ polynomials.

With $f=f\left(x_{1}, x_{2}, \ldots, x_{l}\right)$ be a $\mathbb{C}^{\infty}$ function with multi-variables and $f_{r_{1} x_{1}, \ldots, r_{l} x_{l}}=\partial_{x_{1}}^{r_{1}} \ldots \partial_{x_{l}}^{r_{l}} f$, $f_{0 x_{i}} \equiv f$, where $l$ denotes arbitray integer, then

$$
Y_{n_{1} x_{1}, \ldots, n_{l} x_{l}}(f) \equiv Y_{n_{1}, \ldots, n_{l}}\left(\left\{f_{r_{1} x_{1}, \ldots, r_{l} x_{l}}\left(1 \leq r_{i} \leq n_{i}, 0 \leq i \leq l\right)\right\}\right)=e^{-f} \partial_{x_{1}}^{n_{1}} \ldots \partial_{x_{l}}^{n_{l}} e^{f}
$$

is a polynomial in the partial derivatives of $f$ with respect to $x_{1}, \ldots, x_{l}$, which called the multi-dimensional Bell polynomials.

Based on the multi-dimensional Bell polynomials, the multi-dimensional binary Bell polynomials can be defined as follows:

$$
\mathscr{Y}_{n_{1} x_{1}, \ldots, n_{l} x_{l}}(v, w) \equiv Y_{n_{1} x_{1}, \ldots, n_{l} x_{l}}(f) \equiv Y_{n_{1}, \ldots, n_{l}\left(\left\{f_{\left.\left.r_{1} x_{1}, \ldots, x_{l} x_{l}\right\}\right)}\right\}\right.} \mid f_{r_{r_{1} x_{1}, \ldots, r_{l} x_{l}}=}\left\{\begin{array}{c}
v_{r_{1} x_{1}, \ldots, r_{l} l_{l}}, \sum_{i=1}^{l} r_{i} \text { is odd, } \\
w_{r_{1} x_{1}, \ldots, r_{l} x_{l}}, \sum_{i=1}^{l} r_{i} \text { is even, }
\end{array}\right.
$$

where the vertical line means that the elements on the left-hand side are chosen according to the rule on the right-hand side, $v$ and $w$ are both the $\mathbb{C}^{\infty}$ functions of $\left(x_{1}, x_{2}, \ldots, x_{l}\right)$.

Proposition 1. The relations between the binary Bell polynomials and the standard Hirota D-operators can be given by the identity

$$
\mathscr{Y}_{n_{1} x_{1}, \ldots, n_{l} x_{l}}\left(v=\ln \frac{F}{G}, w=\ln F G\right)=(F G)^{-1} D_{x_{1}}^{n_{1}} \ldots D_{x_{l}}^{n_{l}} F \cdot G,
$$

where $\sum_{i=1}^{l} n_{i} \geq 1$, and Hirota D-operators defined by

$$
D_{x_{1}}^{n_{1}} \ldots D_{x_{l}}^{n_{l}} F \cdot G=\left.\left(\partial_{x_{1}}-\partial_{x_{1}^{\prime}}\right)^{n_{1}} \ldots\left(\partial_{x_{l}}-\partial_{x_{l}^{\prime}}\right)^{n_{l}} F\left(x_{1}, \ldots, x_{l}\right) G\left(x_{1}^{\prime}, \ldots, x_{l}^{\prime}\right)\right|_{x_{1}^{\prime}=x_{1}, \ldots, x_{l}^{\prime}=x_{l}} .
$$


In the particular case of $F=G$, the formula (A.5) can be rewritten as

$$
F^{-2} D_{x_{1}}^{n_{1}} \cdots D_{x_{l}}^{n_{l}} F \cdot F=y_{n_{1} x_{1}, \cdots, n_{l} x_{l}}(0, q=w-v=2 \ln F)=\left\{\begin{array}{l}
0, \sum_{i=1}^{l} n_{i} \text { is odd, } \\
P_{n_{1} x_{1}, \cdots, n_{l} x_{l}}(q), \sum_{i=1}^{l} n_{i} \text { is even, }
\end{array}\right.
$$

which is also called P-polynomials

$$
P_{n_{1} x_{1}, \ldots, n_{l} x_{l}}(q)=\mathcal{Y}_{n_{1} x_{1}, \ldots, n_{l} x_{l}}(0, q=2 \ln F)
$$

where they vanish unless $\sum_{i=1}^{l} n_{i}$ is even.

The binary Bell polynomials $\mathscr{Y}_{n_{1} x_{1}, \ldots, n_{l} x_{l}}(v, w)$ can be written as the combination of $P$-polynomials and $\mathscr{Y}$-polynomials

$$
\begin{aligned}
& (F G)^{-1} D_{x_{1}}^{n_{1}} \ldots D_{x_{l}}^{n_{l}} F \cdot G \\
& =\left.\mathscr{Y}_{n_{1} x_{1}, \ldots, n_{l} x_{l}}(v, w)\right|_{v=\ln F / G, w=\ln F G} \\
& =\left.\mathscr{Y}_{n_{1} x_{1}, \ldots, n_{l} x_{l}}(v, v+q)\right|_{v=\ln F / G, q=2 \ln G} \\
& =\sum_{r_{1}=0}^{n_{1}} \ldots \sum_{r_{l}=0}^{n_{l}} \prod_{i=1}^{l}\left(\begin{array}{c}
n_{i} \\
r_{i}
\end{array}\right) P_{n_{1} x_{1}, \ldots, n_{l} x_{l}}(q) Y_{\left(n_{1}-r_{1}\right) x_{1}, \ldots,\left(n_{l}-r_{l}\right) x_{l}}(v) .
\end{aligned}
$$

Proposition 2. Under the Hopf-Cole transformation $v=\ln \psi$, i.e., $\psi=F / G$, the $\mathscr{Y}$-polynomials can be written as

$$
\left.Y_{n_{1} x_{1}, \ldots, n_{l} x_{l}}(v)\right|_{v=\ln \psi}=\frac{\psi_{n_{1} x_{1}, \ldots, n_{l} x_{l}}}{\psi},
$$

which provides the shortest way to the associated Lax systems of NLEEs.

\section{Appendix B. Riemann theta function}

Based on the results in Ref. [23, 27], when $N=1$, the Riemann theta function reduces the following Fourier series in $n$ :

$$
\vartheta(\theta, \tau)=\sum_{n=-\infty}^{+\infty} e^{\pi i n^{2} \tau+2 \pi i n \theta},
$$

which can be used to construct the one periodic solution and the phase variable $\theta=a_{1} x_{1}+a_{2} x_{2}+\ldots+a_{j} x_{j}+a_{0}$ and the parameter $\operatorname{Im}(\tau)>0$.

Theorem 8. Assuming that $\vartheta(\theta, \tau)$ is a Riemann theta function for $N=1$ with $\theta=a_{1} x_{1}+a_{2} x_{2}+\ldots+a_{j} x_{j}+a_{0}$ and the parameters $a_{1}, a_{2}, \ldots, a_{j}, a_{0}$ satisfy the following system

$$
\begin{aligned}
& \sum_{n=-\infty}^{+\infty} \mathscr{L}\left[4 n \pi i a_{1}, 4 n \pi i a_{2}, \ldots, 4 n \pi i a_{j}, 4 n \pi i a_{0}\right] e^{2 n^{2} \pi i \tau}=0, \\
& \sum_{n=-\infty}^{+\infty} \mathscr{L}\left[2 \pi i(2 n-1) a_{1}, 2 \pi i(2 n-1) a_{2}, \ldots, 2 \pi i(2 n-1) a_{j}, 2 \pi i(2 n-1) a_{0}\right] e^{\left(2 n^{2}-2 n+1\right) \pi i \tau}=0,
\end{aligned}
$$


the expression

$$
u=h \partial_{x_{j}}^{p} \ln \vartheta(\theta, \tau)
$$

is the one periodic wave solution of the NLEE.

We write the coefficient matrix and the vector of system (6.9) into power series of $\delta$

$$
\begin{aligned}
& \left(\begin{array}{ll}
a_{11} & a_{12} \\
a_{21} & a_{22}
\end{array}\right)=A_{0}+A_{1} \delta+A_{2} \delta^{2}+\cdots, \\
& \left(\begin{array}{c}
-b_{1} \\
-b_{2}
\end{array}\right)=B_{0}+B_{1} \delta+B_{2} \delta^{2}+\cdots, \\
& \left(\begin{array}{c}
\omega \\
-c
\end{array}\right)=X_{0}+X_{1} \delta+X_{2} \delta^{2}+\cdots
\end{aligned}
$$

where $\delta=e^{\pi i \tau}$.

Substituting (B.4) into (6.9), we have the following recursion relations

$$
\begin{aligned}
& A_{0} X_{0}=B_{0}, \\
& A_{0} X_{1}+A_{1} X_{0}=B_{1}, \\
& A_{0} X_{2}+A_{2} X_{0}+A_{1} X_{1}=B_{2}, \\
& \ldots, \\
& A_{0} X_{n}+A_{1} X_{n-1}+\ldots+A_{n} X_{0}=B_{n} .
\end{aligned}
$$

Theorem 9. If the matrix $A_{0}$ is reversible, solving (B.5) leads to

$$
X_{0}=A_{0}^{-1} B_{0}, X_{n}=A_{0}^{-1}\left(B_{n}-\sum_{j=1}^{n} A_{j} B_{n-1}\right), n=1,2, \ldots
$$

If $A_{0}$ and $A_{1}$ are not inverse, but they take the following form

$$
A_{0}=\left(\begin{array}{ll}
0 & 1 \\
0 & 0
\end{array}\right), A_{1}=\left(\begin{array}{cc}
0 & 0 \\
-8 \pi^{2} \rho & 2
\end{array}\right),
$$


solving relations (B.5) leads to

$$
\begin{aligned}
& X_{0}=\left(\begin{array}{c}
-\frac{1}{8 \pi^{2} \rho}\left(B_{1}^{(I I)}-2 B_{0}^{(I)}\right) \\
B_{0}^{(I)}
\end{array}\right), \\
& X_{1}=\left(\begin{array}{c}
-\frac{1}{8 \pi^{2} \rho}\left[\left(B_{2}-A_{2} X_{0}\right)^{(I I)}-2 B_{1}^{(I)}\right] \\
B_{1}^{(I)}
\end{array}\right), \\
& \ldots, \\
& X_{n}=\left(\begin{array}{c}
-\frac{1}{8 \pi^{2} \rho}\left[\left(B_{n+1}-\sum_{j=2}^{n+1} A_{j} X_{n+1-j}\right)_{1}^{(I I)}-2\left(B_{n+1}-\sum_{j=2}^{n} A_{j} X_{n-j}\right)^{(I)}\right] \\
\left(B_{n+1}-\sum_{j=2}^{n} A_{j} X_{n-j}\right)^{(I)}
\end{array}\right), n=2,3, \ldots
\end{aligned}
$$

where $V^{I}$ and $V^{I I}$ denote the first and second component of a two dimensional vector $V$, respectively.

\section{References}

\section{References}

[1] R. Hirota, Direct Methods in Soliton Theory, Springer-verlag, Berlin, 2004.

[2] J. Hietarinta, A. Ramani, B. Grammaticos, Continuous vacua in bilinear soliton equations, J. Phys. A: Math. Gen. 27 (1994) $3149-3158$.

[3] X.B. Hu, Y.T. Wu, S.M. Zhu, X.G. Geng, Some new results on the triple-humped soliton equation: Bäcklund transformation, superposition principle and new explicit solutions, J Phys A: Math Gen 31 (1998) 8859-8868.

[4] Q.P. Liu, X.B. Hu, M.X. Zhang, Supersymmetric modified Korteweg-de Vries equation: bilinear approach, Nonlinearity 18 (2005) $1597-$ 1603.

[5] Alvaro H. Salas, Computing solutions to a forced KdV equation, Nonlinear Anal.: Real 12(2) (2011) 1314-1320.

[6] S.F. Shen, Y.Y. Jin, Some new soliton equations with self-consistent sources, Nonlinear Anal.: Real 12(2) (2011) 895-901.

[7] E. T. Bell, Exponential polynomials, Ann. Math. 35 (1934) 258-277.

[8] G. Gilson, F. Lambert, J. Nimmo, R. Willox, On the combinatorics of the Hirota D-operators, Proc. R. Soc. Lond. A 452 (1996) $223-234$.

[9] F. Lambert, I. Loris, J. Springael, Classical Darboux transformations and the KP hierarchy, Inverse Probl. 17 (2001) 1067-1074.

[10] Y.H. Wang, Y. Chen, Binary Bell polynomial manipulations on the integrability of a generalized (2+1)-dimensional Korteweg-de Vries equation, J. Math. Anal. Appl. 400 (2013) 624-634.

[11] Y.H. Wang, Y. Chen, Integrability of the modified generalised Vakhnenko equation, J. Math. Phys. 53 (2012) 123504.

[12] E.G. Fan, The integrability of nonisospectral and variable-coefficient KdV equation with binary Bell polynomials, Phys. Lett. A 375 (2011) 493-497.

[13] Y.C. Hon, E.G. Fan, Binary Bell polynomial approach to the non-isospectral and variable-coefficient KP equations, IMA J. Appl. Math. 77(2) (2012) 236-251.

[14] E.G. Fan, New bilinear Bäcklund transformation and Lax pair for the supersymmetric Two-Boson equation, Stud. Appl. Math. 127 (2011) 284-301.

[15] E.G. Fan, Y.C. Hon, Super extension of Bell polynomials with applications to supersymmetric equations, J. Math. Phys. 53 (2012) 013503.

[16] W.X. Ma, Bilinear equations, Bell polynomials and linear superposition principle, J Phys: Conf. Ser. 411 (2013) 012021.

[17] T.R. Marchant, Solitary wave interaction for the extended BBM equation, Proc. R. Soc. Lond. A 456 (2000) 433-453. 
[18] T.R. Marchant, Numerical solitary wave interaction: the order of the inelastic effect, ANZIAM J. 44 (2002) 95-102.

[19] A.M. Wazwaz, Partial Differential Equations and Solitary Waves Theory, Higher Education Press, Beijing, 2009.

[20] A. Nakamura, A direct method of calculating periodic wave solutions to nonlinear evolution equations. I. exact two-periodic wave solution, J. Phys. Soc. Jpn. 47 (1979) 1701-1705.

[21] A. Nakamura, A direct method of calculating periodic wave solutions to nonlinear evolution equations. II. exact one-and two-periodic wave solution of the coupled bilinear equations, J. Phys. Soc. Jpn. 48 (1980) 1365-1370.

[22] H.H. Dai, E.G. Fan, X.G. Geng, Constructing periodic wave solutions of nonlinear equations by Hirota bilinear method, arXiv:nlin/0602015, 2006.

[23] E.G. Fan, Y.C. Hou, Quasiperiodic waves and asymptotic behavior for Bogoyavlenskii's breaking soliton equation in (2+1) dimensions, Phys. Rev. E 78 (2008) 036607.

[24] E.G. Fan, K.W. Chow, On the periodic solutions for both nonlinear differential and difference equations: A unified approach, Phys. Lett. A 374 (2010) 3629-3634.

[25] Y. Zhang, L.Y. Ye, Y.N. Lv, H.Q. Zhao, Periodic wave solutions of the Boussinesq equation, J. Phys. A: Math. Theor. 40 (2007) $5539-5549$.

[26] W.X. Ma, R.G. Zhou, L. Gao, Exact one-periodic and two-periodic wave solutions to Hirota bilinear equations in (2+1) dimensions, Mod. Phys. Lett. A 24 (2009) 1677-1688.

[27] S.F. Tian, H.Q. Zhang, Riemann theta functions periodic wave solutions and rational characteristics for the nonlinear equations, J. Math. Anal. Appl. 371 (2010) 585-608. 


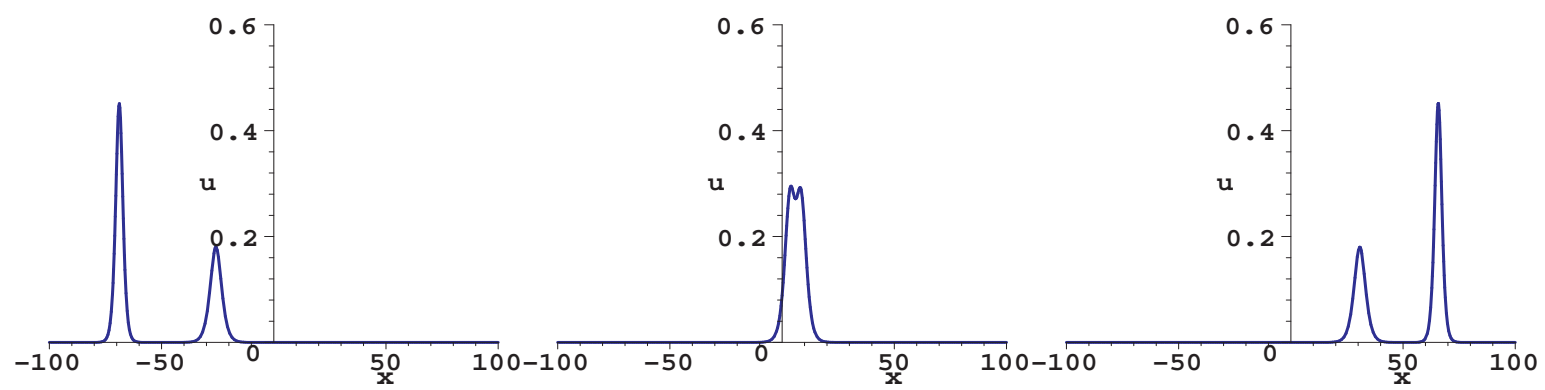
(a) $\mathrm{t}=-20$
(b) $\mathrm{t}=2.3$
(c) $\mathrm{t}=20$

Figure B.1: Overtaking collision of two solitons via solution (2.14). Parameters are $k_{1}=0.95, k_{2}=-0.6, \alpha=0.8, c_{4}=3$ and $\zeta_{1}=\zeta_{2}=0$.

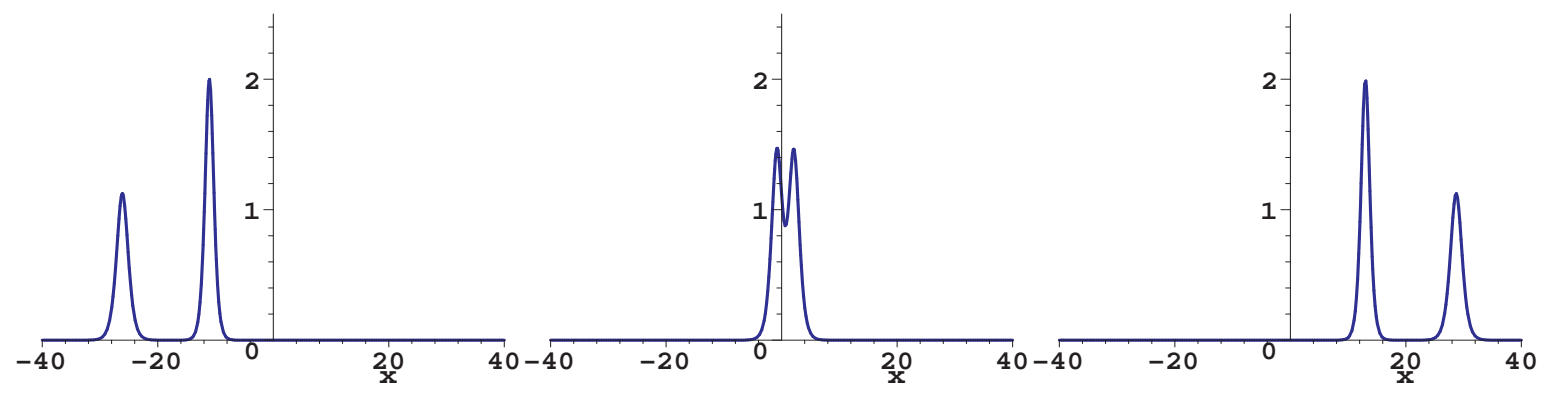
(a) $\mathrm{t}=-25$
(b) $\mathrm{t}=-0.6$
(c) $\mathrm{t}=25$

Figure B.2: Overtaking collision of two solitons via solution (2.14). Parameters are $k_{1}=1.5, k_{2}=2, \alpha=0.2, c_{4}=-2$ and $\zeta_{1}=\zeta_{2}=0$.

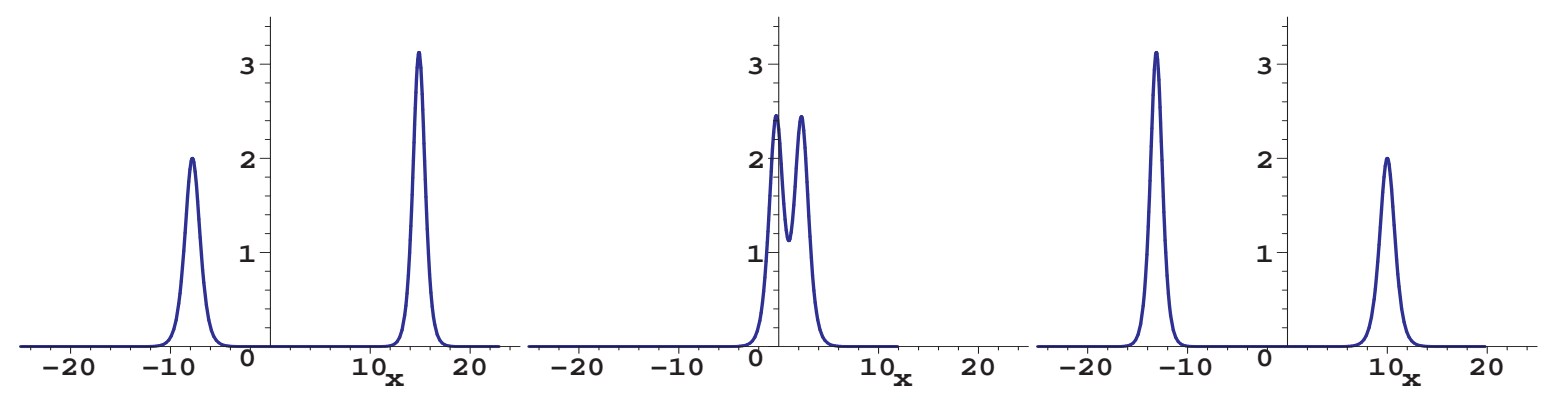
(a) $\mathrm{t}=-15$
(b) $\mathrm{t}=-0.15$
(c) $\mathrm{t}=15$

Figure B.3: Head-on collision of two solitons via solution (2.14). Parameters are $k_{1}=2.5, k_{2}=2, \alpha=0.2, c_{4}=-2$ and $\zeta_{1}=\zeta_{2}=0$. 


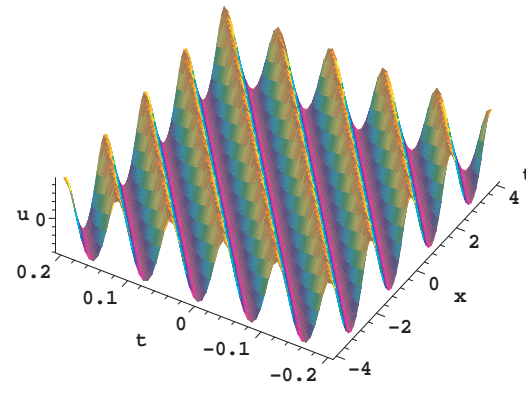

(a)

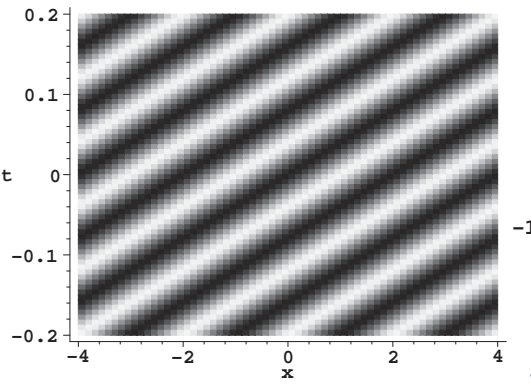

(b)

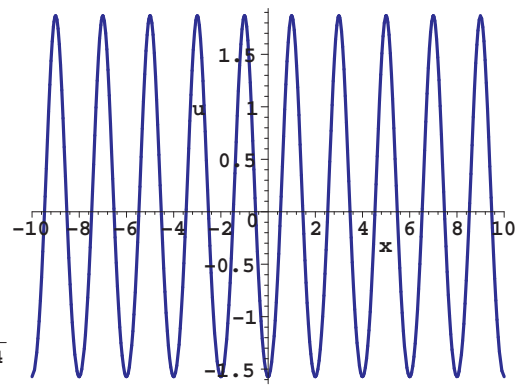

(c)

Figure B.4: A one periodic wave of the Sawada-Kotera-type equation (1.2) via expression $(6.10)$ with the parameters $c_{1}=45 c_{4}, c_{2}=c_{3}=$ $15 c_{4}, c_{4}=1, \alpha=1, \gamma_{1}=0, \rho_{1}=0.5$. (a) The perspective view of the real part of the periodic wave. (b) Overhead view of the wave, with contour plot shown. The bright lines are crests and the dark lines are troughs. (c) The wave propagation pattern of the wave along the $x$ axis. 


\section{Figure captions}

1. Fig. B.1: Overtaking collision of two solitons via solution (2.14). Parameters are $k_{1}=0.95, k_{2}=-0.6, \alpha=$ $0.8, c_{4}=3$ and $\zeta_{1}=\zeta_{2}=0$.

2. Fig. B.2: Overtaking collision of two solitons via solution (2.14). Parameters are $k_{1}=1.5, k_{2}=2, \alpha=0.2, c_{4}=$ -2 and $\zeta_{1}=\zeta_{2}=0$.

3. Fig. B.3: Head-on collision of two solitons via solution (2.14). Parameters are $k_{1}=2.5, k_{2}=2, \alpha=0.2, c_{4}=$ -2 and $\zeta_{1}=\zeta_{2}=0$.

4. Fig. B.4: A one periodic wave of the Sawada-Kotera-type equation (1.2) via expression (6.10) with the parameters $c_{1}=45 c_{4}, c_{2}=c_{3}=15 c_{4}, c_{4}=1, \alpha=1, \gamma_{1}=0, \rho_{1}=0.5$. (a) The perspective view of the real part of the periodic wave. (b) Overhead view of the wave, with contour plot shown. The bright lines are crests and the dark lines are troughs. (c) The wave propagation pattern of the wave along the $x$ axis. 